\title{
Recurrent dermatofibrosarcoma protuberans of head and neck with fibrosarcomatous change: a challenge for the head and neck surgeon
}

\author{
Balaji Ramamourthy', Kanika Arora ${ }^{1}$, Balamurugan Thirunavukkarasu², \\ Anurag S. Ramavat ${ }^{1 *}$, Amanjit Bal ${ }^{2}$
}

\begin{abstract}
${ }^{1}$ Department of Otolaryngology, Head and Neck Surgery, ${ }^{2}$ Department of Histopathology, PGIMER, Chandigarh, India
\end{abstract}

Received: 01 June 2020

Accepted: 04 July 2020

\section{*Correspondence:}

Dr. Anurag S. Ramavat,

E-mail: ramavatanu@gmail.com

Copyright: (C) the author(s), publisher and licensee Medip Academy. This is an open-access article distributed under the terms of the Creative Commons Attribution Non-Commercial License, which permits unrestricted non-commercial use, distribution, and reproduction in any medium, provided the original work is properly cited.

\begin{abstract}
Dermatofibrosarcoma protuberans (DFSP) of head and neck is a rare, slow growing, intermediate to low grade malignant soft tissue neoplasm with high rates of local recurrence requiring multiple surgeries posing great distress for both the patient and treating physician alike. Fibrasarcomatous change is a very rare occurrence in such tumors increasing risk of distant metastasis. We report such a rare case of recurrent DFSP with fibrosarcomatous change of the head and neck region, the management protocol and the key points a surgeon should keep in mind while treating such patients.
\end{abstract}

Keywords: Dermatofibrosarcoma protuberans, Head and neck, Recurrent, Fibrosarcoma

\section{INTRODUCTION}

Dermatofibrosarcoma protuberans (DFSP) is a rare locally aggressive mesenchymal tumor of fibroblast origin; it accounts for less than $1 \%$ of all soft tissue sarcomas and $<0.1 \%$ of all malignancies. ${ }^{1}$ Taylor recognised it for the first time in $1890 .^{2}$ Darrier was the first to describe it in $1924 .^{3}$ Hoffman coined the term "dermatofibrosarcoma protuberans" in $1925 .{ }^{4}$ It most commonly involves the trunk, followed by the upper and lower extremities, and head and neck. ${ }^{5}$ Fibrosarcomatous change is a rare occurrence and has a higher rate of recurrence and metastasis. We report such a rare case of head and neck recurrent DFSP with fibrosarcomatous transformation and challenges faced by head and neck surgeons treating them.

\section{CASE REPORT}

A 28 years old female patient presented with complaints of recurrent swelling in post auricular region, which was insidious in onset and gradually progressive over 6 months duration. The swelling was painless and not associated with dysphagia dyspnea change in voice, facial weakness or ear complaints. Patient had similar swelling in the same location in the past and was operated elsewhere twice.

On examination the lesion was $15 \times 9 \mathrm{~cm}$ in size situated in the right side post auricular region with irregular bosselated surface and prominent vessels and causing thinning of overlying skin (Figure 1). The ventral surface of pinna was involved by the disease and pushed forward. The swelling had a firm consistency non-tender nonpulsatile not compressible with restricted mobility in both vertical and horizontal direction. Facial nerve was intact. No intraoral bulge was present and parotid duct was normal. There were no cervical lymph nodes palpable.

The patient underwent contrast enhanced magnetic resonance imaging scan, which showed heterogeneously hyper-intense lesion on T2 and hypo-intense on T1 with 
multiple non-enhancing areas. The lesion was involving the trapezius muscle and was closely abutting sternocleidomastoid and superficial lobe of parotid gland.

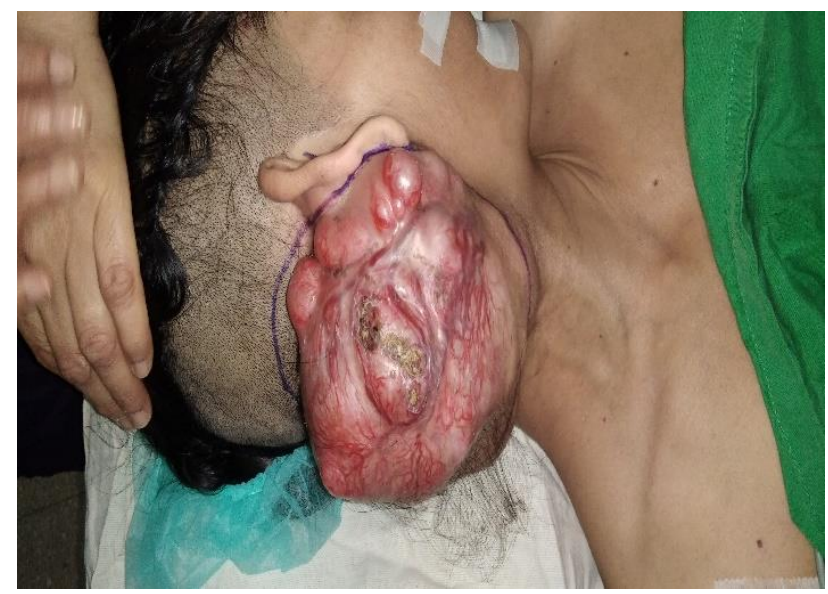

Figure 1: $15 \times 9 \times 8 \mathrm{~cm}$ tumor in post-auricular region with nodular surface and prominent vessels. Resection margin of $2 \mathrm{~cm}$ marked.

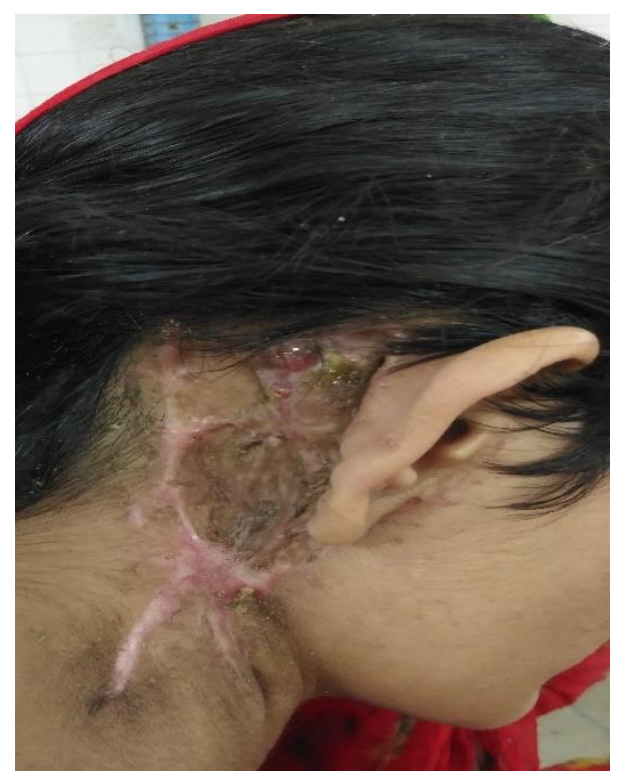

Figure 2: Post-operative wound at one month follow up. Well healed split skin graft.

Computed tomography with angiography (prominent vessels over the swelling) showed arterial feeders from right occipital artery and V2/V3 segment of vertebral artery. Mastoid cortex was found eroded.

Patient underwent wide local excision with selective neck dissection of levels 2-4 under general anesthesia.

A resection margin of $2 \mathrm{~cm}$ was included with the specimen and the eroded part of mastoid bone was drilled out. The conchal cartilage was removed and subcentimetric nodes from level 2-4 were removed and sent for pathological examination. Facial nerve was identified and preserved. The defect was covered with split thickness skin graft (Figure 2).

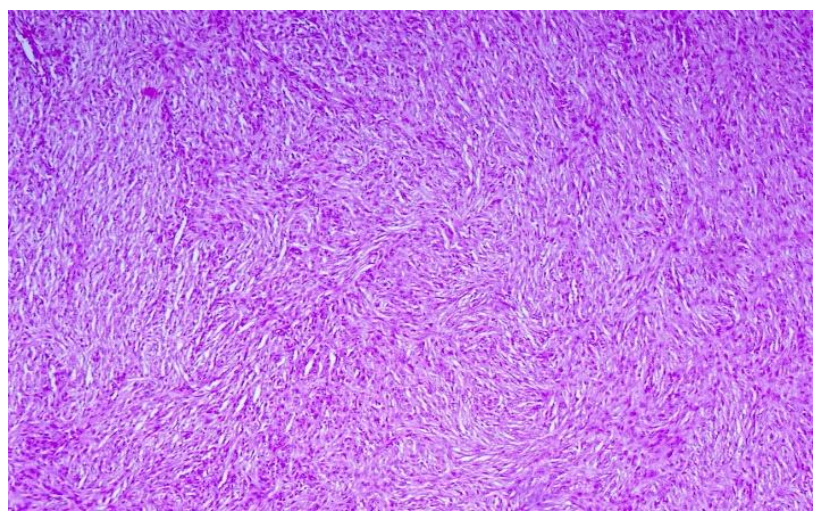

Figure 3: Uniform population of spindled tumor cells (fibroblasts) arranged in a distinct, monotonous, "storiform" pattern around an inconspicuous vasculature. There is minimal nuclear pleomorphism and only low mitotic activity (H\&E 100X).

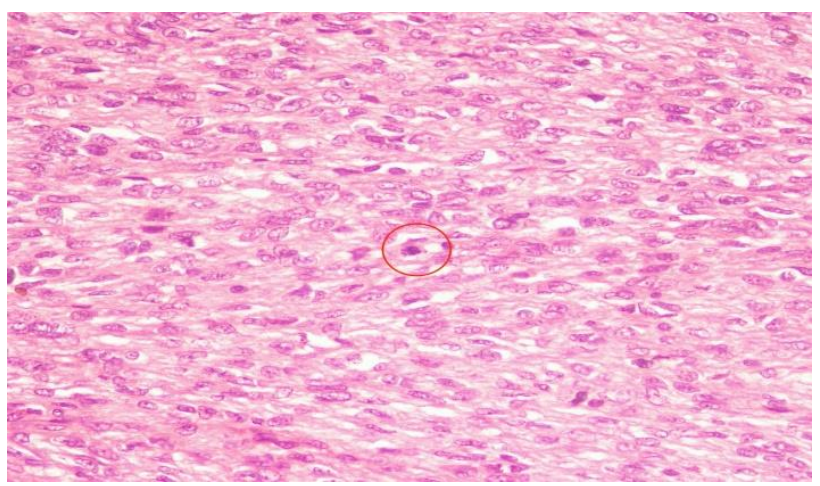

Figure 4: Areas with fibrosarcomatous transformation where the tumor cells are arranged in fascicular architecture and herring bone pattern (loss of storiform pattern) with moderate nuclear pleomorphism and increased mitotic activity (within red circle) (H\&E 400X).

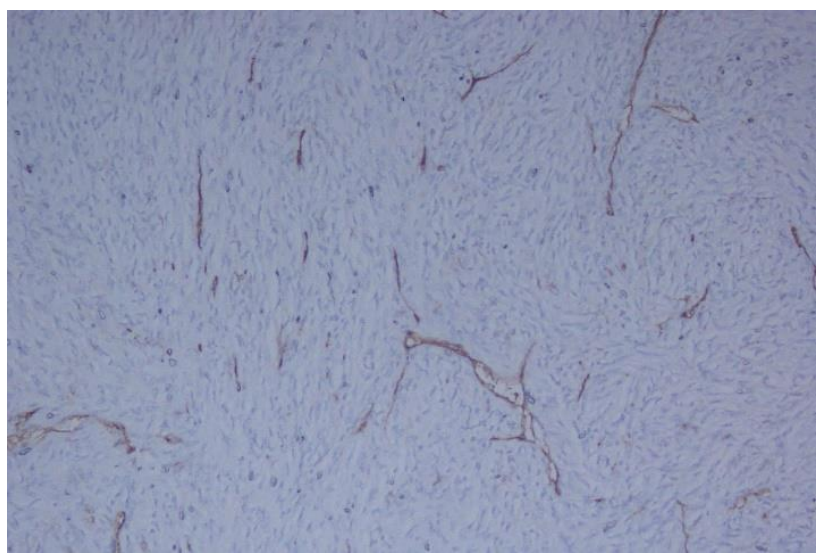

Figure 5: Tumor cells show loss of CD34 in the fibrosarcomatous areas (vessels normal control) (immunoperoxidase 400X). 
Histological examination showed circumscribed tumor involving dermal and subcutaneous layer with tumor cells arranged in long fascicles and focal storiform pattern (Figure 3). It also showed areas of increased cellularity with high-grade features and mitosis (Figure 4). The tumor cells were elongated with coarse chromatin, inconspicuous nucleoli and moderate amount of cytoplasm. Immunohistochemistry showed strong CD34 positivity with areas of loss of CD34 (Figure 5). The resection margins were free of tumor and lymph nodes were free of tumor. The features were of DFSP with fibrosarcomatous transformation.

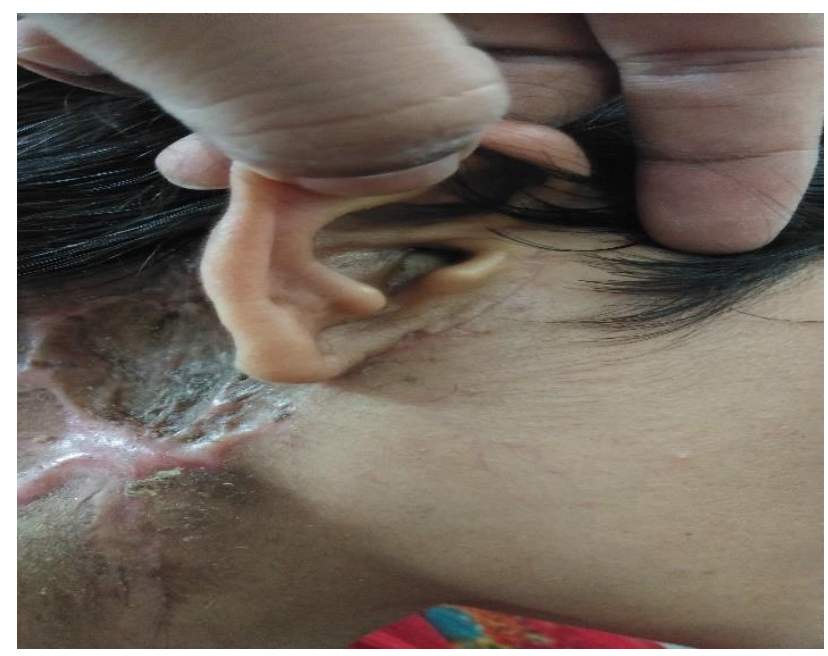

Figure 6: Partial stenosis of external auditory canal.

Post operatively the patient was subjected to adjuvant radiotherapy because of its recurrent course and fibrosarcomatous change. Patient was followed up for a period of one year and was found asymptomatic with no recurrence. However, patient developed partial stenosis of external auditory canal as a complication as conchal cartilage was removed (Figure 6).

\section{DISCUSSION}

DFSP is a rare, low-grade sarcoma of dermal fibroblast origin with an incidence of 4.2-4.5 cases per million persons per year. ${ }^{6}$ Its has an indolent course, with very high recurrence rate because of its propensity to infiltrate subcutaneous tissue, fascia and underlying muscle in the form of pseudopods. ${ }^{7,8}$ DFSP usually affects individuals aged between 20 and 50 years and females more than males. ${ }^{9}$

The tumor begins as a violaceous reddish-brown or pink firm to hard indolent plaque and later infiltrates the deep layers of the skin and the subcutaneous tissue, leading to development of multiple nodules, which are indurated on palpation and adherent to the adjoining structures like the subcutaneous fat, fascia, muscle, periosteum and bone. ${ }^{10}$ Our patient had a huge lesion with attachment to underlying trapezius muscle and erosion of temporal bone.
Chromosomal translocation $\mathrm{t}(17 ; 22)(\mathrm{q} 22 ; \mathrm{q} 13)$ between chromosomes 17 and 22 is thought to be a key in the tumor's pathogenesis. This leads to the fusion of plateletderived growth factor (PDGF) gene and collagen type 1A1 gene causing overproduction of PDGF causing cellular proliferation and tumor formation. This translocation is present in over $90 \%$ of DFSP. ${ }^{11}$

The treatment of DFSP is essentially surgical. Wide local surgical resection with recommended surgical margins of $2-3 \mathrm{~cm}$ and three-dimensional resection is the standard treatment for a localized disease. ${ }^{12}$

Inadequate surgical margins leads to higher recurrence rates. ${ }^{13}$ Even with $3 \mathrm{~cm}$ surgical margins a recurrence rate of $3 \%$ has been described. ${ }^{14}$ DFSP of head and neck are even more challenging because of the risk of disfigurement and functional impairment. Moh's micrographic surgery has lower recurrence rate and is a better option for DFSP of Head and neck. ${ }^{13,15}$ As the facility for Moh's micrographic surgery was not available we proceeded with wide local excision with resection margin of $2 \mathrm{~cm}$.

Microscopically it is characterized by the arrangement of spindle-shaped tumor cells in a "cartwheel" or "storiform" pattern. Cytologically monomorhous spindle cells, with a typical finger-like, honeycomb pattern of infiltration into the subcutaneous plane, which may eject up to $3 \mathrm{~cm}$ peripherically. ${ }^{16}$

Immunohistochemical staining shows strong positivity for CD34 and vimentin and negativity for factor XIIIa, S100 and CD44 staining. ${ }^{17}$ An aggressive subtype presents with fibrosarcomatous progression is called "fibrosarcomatous dermatofibrosarcoma" (FS-DFSP). Penner in 1951 reported it for the first time. It is an intermediate to high-grade sarcoma, with greater number of nuclei and increased mitotic rate and more spindle cells compared to "classic" DFSP. Immunohistochemically has a decrease of CD34. ${ }^{18}$ It is rare and appears in $10-15 \%$ of DFSP cases, characterized by a higher incidence of local relapse and distance metastasis. According to various authors progression of DFSP to FSDFSP may represent "dedifferentiation" or a form of tumor progression with increased risk of metastasis, associated with gains of p53 mutations and increased proliferation. ${ }^{19,20}$

The head and neck region has the highest rate of local recurrence rate (LRR) after wide local excision. Barnes et al reported $53 \%$ and $73 \%$ LRR in two studies. ${ }^{21}$ Mark et al also reported very high LRR $(60 \%)$ in HN-DFSP. ${ }^{22}$ Head and neck surgeons are more conservative in excision as critical structures are involved in the area and reconstructive difficulties of the surgical defect. Multiple recurrences due to inadequate control of the initial disease predisposes to distant metastasis and poor outcome..$^{23}$ 
FS-DFSP subtype is also considered to be a significant prognostic factor for relapse. The 5-year recurrence free survival rate was $81 \%$ for patients with DFSP and only $28 \%$ for those with FS-DFSP subtype. ${ }^{24}$ In Mentzel's study, follow up of 34 from 41 FS-DFSP patients revealed local relapse in 20 patients $(58 \%) .{ }^{19}$

Radiotherapy has an adjunctive role in treatment as the tumor has been shown to be radiosensitive; chemotherapy with selective tyrosine kinase inhibitory such as imatinib mesylate has also shown good results. ${ }^{25}$ However, radiotherapy is to be considered in patients with local recurrence or positive or close margins on surgery. In our patient post-operative resection margins were all free however, due to the recurrent nature and fibrosarcomatous changes patient was subjected to postoperative radiotherapy. Patient is disease free 12 months post operatively and is on regular follow up.

\section{CONCLUSION}

Head and neck DFSP, a benign condition poses lot of challenges for a head and neck surgeon. Its high recurrence rates because of the pseudopodia, cosmetic problems due to excision, reconstructive difficulties include few of them. Wide resection margin of 3-5 cm or Moh's micrographic surgery reduces the recurrence rate. Multidisciplinary team approach will help in reconstruction of huge defects. Adjuvant therapy with radiotherapy and imatinib in select cases will help in preventing local cases and in palliative set up. Long-term follow up is essential in all patients.

\section{Funding: No funding sources}

Conflict of interest: None declared

Ethical approval: Not required

\section{REFERENCES}

1. Llombart B, Serra-Guillén C, Monteagudo C, Guerrero JA, Sanmartín O. Dermatofibrosarcoma protuberans: a comprehensive review and update on diagnosis and management. In Seminars in Diagnostic Pathol. 2013;30(1):13-28.

2. Taylor RW. Sarcomatous tumors resembling in some respects keloids. Arch Dermatol. 1890;8:3847.

3. Darier J. Dermatofibromes progressifs et recidivants ou fibrosarcomes de la peau. Ann Dermatol Venereol. 1924;5:545-62.

4. Hoffmann EI. Über das knollentreibende Fibrosarkom der Haut (Dermatofibrosarkoma protuberans). Dermatology. 1925;43(1-2):1-28.

5. Kim BJ, Kim H, Jin US, Minn KW, Chang H. Wide local excision for dermatofibrosarcoma protuberans: a single-center series of 90 patients. BioMed Res Int. 2015;2015.

6. National Comprehensive Cancer Network. NCCN Clinical Practice Guidelines in Oncology Dermatofibrosarcoma Protuberans; 2017.
7. Pérez Olga G, Rodolfo R, Alberto W, Hugo A. Make your diagnosis. An. Bras. Dermatol. 2010;85(2):245-7.

8. Criscione VD, Weinstock MA. Descriptive epidemiology of dermatofibrosarcoma protuberans in the United States, 1973 to 2002. J Am Acad Dermatol. 2007;56(6):968-73.

9. Sanmartín O, Llombart B, López-Guerrero JA, Serra C, Requena C, Guillén C. Dermatofibrosarcoma protuberans. Actas DermoSifiliograficas. 2007;98(2):77-87.

10. Stivala A, Lombardo GA, Pompili G, Tarico MS, Fraggetta F, Perrotta RE. Dermatofibrosarcoma protuberans: Our experience of 59 cases. Oncol Letters. 2012;4(5):1047-55.

11. Brooks J, Ramsey ML. Cancer, dermatofibrosarcoma protuberans. In Stat Pearls. 2019.

12. Kimmel Z, Ratner D, Kim JY, Wayne JD, Rademaker AW, Alam M. Peripheral excision margins for dermatofibrosarcoma protuberans: a meta-analysis of spatial data. Ann Surg Oncol. 2007;14(7):2113-20.

13. Lemm D, Mügge LO, Mentzel T, Höffken K. Current treatment options in dermatofibrosarcoma protuberans. J Cancer Res Clin Oncol. 2009; 135(5):653-65.

14. Thomas CJ, Wood GC, Marks VJ. Mohs micrographic surgery in the treatment of rare aggressive cutaneous tumors: the Geisinger experience. Dermatol Surg. 2007;33(3):333-9.

15. Paradisi A, Abeni D, Rusciani A, Cigna E, Wolter M, Scuderi N, Rusciani L, Kaufmann R, Podda M. Dermatofibrosarcoma protuberans: wide local excision vs. Mohs micrographic surgery. Cancer Treatment Rev. 2008;34(8):728-36.

16. Taylor HB, Helwig EB. Dermatofibrosarcoma protuberans. A study of 115 cases. Cancer. 1962;15(4):717-25.

17. Maggoudi D, Vahtsevanos K, Psomaderis K, Kiesaridou D, Valery R, Karakinaris G. Dermatofibrosarcoma protuberans of the face: report of 2 cases and an overview of the recent literature. J Oral Maxillofacial Surg. 2006;64(1):140-4.

18. Penner DW. Metastasizing dermatofibrosarcoma protuberans. A case report. Cancer. 1951;4(5):10836.

19. Mentzel T, Beham A, Katenkamp D, Dei Tos AP, Fletcher CD. Fibrosarcomatous ("high-grade") dermatofibrosarcoma protuberans: clinicopathologic and immunohistochemical study of a series of 41 cases with emphasis on prognostic significance. Am J Surg Patho. 1998;22(5):576-87.

20. Abbott JJ, Oliveira AM, Nascimento AG. The prognostic significance of fibrosarcomatous transformation in dermatofibrosarcoma protuberans. Am J Surg Pathol. 2006;30(4):436-43. 
21. Barnes L, Coleman JA, Johnson JT. Dermatofibrosarcoma protuberans of the head and neck. Arch Otolaryngol. 1984;110(6):398-404.

22. Mark RJ, Bailet JW, Tran LM, Poen J, Fu YS, Calcaterra TC. Dermatofibrosarcoma protuberans of the head and neck: a report of 16 cases. Arch Otolaryngol Head Neck Surg. 1993;119(8):891-6.

23. Rutgers EJ, Kroon BB, Albus-Lutter CE, Gortzak E. Dermatofibrosarcoma protuberans: treatment and prognosis. Eur J Surg Oncol. 1992;18(3):241-8.

24. Bowne WB, Antonescu CR, Leung DH, Katz SC, Hawkins WG, Woodruff JM, et al. Dermatofibrosarcoma protuberans: a clinicopathologic analysis of patients treated and followed at a single institution. Cancer. 2000;88(12):2711-20.

25. Angouridakis N, Kafas P, Jerjes W, Triaridis S, Upile T, Karkavelas G, et al. Dermatofibrosarcoma protuberans with fibrosarcomatous transformation of the head and neck. Head Neck Oncol. 2011;3(1):5.

Cite this article as: Ramamourthy $\mathrm{B}$, Arora K, Thirunavukkarasu B, Ramavat AS, Bal A. Recurrent dermatofibrosarcoma protuberans of head and neck with fibrosarcomatous change: a challenge for the head and neck surgeon. Int J Otorhinolaryngol Head Neck Surg 2020;6:1563-7. 